\title{
A Case Study of Natural Frequency of the Tram Rail Due to Vibration Using Wavelets
}

\author{
Pushan Kumar Dutta*1, Mircea Bogdan Tătaru ${ }^{2}$, Ovidiu Moldovan ${ }^{3}$, Tiberiu Vesselenyi ${ }^{4}$ \\ ${ }^{1}$ Department of Electronics and Communication Engineering, Rajeev Gandhi Memorial College of Engineering and \\ Technology, India \\ ${ }^{1,2,3}$ Department of Mechatronics, University of Oradea, Oradea, Romania \\ ${ }^{4}$ Department of Mechatronics and Head of Doctoral School, University of Oradea, Oradea, Romania
}

\begin{tabular}{l}
\hline \hline Article Info \\
\hline Article history: \\
Received Dec 9, 2017 \\
Revised Jan 10, 2018 \\
Accepted Jan 27, 2018 \\
\hline
\end{tabular}

Keyword:

Estimation of unevenly sampled signals

Frequency decomposition

Time-frequency contour

Tram Signal Monitoring

wavelet

\begin{abstract}
Many vibration signals of tram rails due to tram movement are nonstationary and have highly complex time-frequency characteristics. The vibration signal of a rotating wheel involves condition monitoring and fault diagnosis. Many signal analysis methods are able to extract useful information from vibration data. In this paper, we were able to correlate nonlinear independent signal acquired using acceleromets at different spots across the city and extract tram rail vibration noise and model the effect of signal noise to identify the frequency characteristics of the rail by characterizing the spectral content of the noise signal using parametric distribution and then by applying non parametric filters to characterize the signal power spectral density using Wavelet Transform (WT) and Parseval's theorem. The fault can be detected from a given level of resolution. For this purpose, Parseval's theorem is used as an evaluation criterion to select the optimal level. Associated to envelope analysis, it allows clear visualization of fault frequencies. on the inner rail of the railway line. The time-frequency contour map can easily show the power distribution of signal in time and frequency domain. Moreover, it is a good way to identify the rail track faults involving a breakdown change. The simulative results show that timefrequency contour map have the capabilities to identify the difference of those faults of vibration monitoring. In conclusion, the faults along the rail track can be classified by time-frequency contour map for frequency decomposition. We hereby decompose the high frequency detail of the signal without decomposition after wavelet transform, so as to improve the frequency resolution.
\end{abstract}

Copyright $@ 2018$ Institute of Advanced Engineering and Science. All rights reserved.

\section{Corresponding Author:}

Pushan Kumar Dutta,

Department of Electronics and Communication Engineering,

Rajeev Gandhi Memorial College of Engineering and Technology, India.

Email: ascendent1@gmail.com

\section{INTRODUCTION}

Acquiring the signal data using accelerometer sensors conducted as in Phase II project in the present scenario and so data has been acquired from Project conducted earlier for 7 locations along Oradea rail tracks along a number of measurement points under Phase II project as shown in Figure 1. Physical deterioration of the tram tracks due to continuous and regular movement of trams is a very critical component of modern Urban transport in Europe. The vibration signal is application dependant and we can say that the process of generation and coupling with system components are done using feature extraction in wavelet domain. There are many ways to analyze it in different decompose sub level as Kurtosis, frequency signature in deferent level, singularities etc. The frequency in decomposing sub signal depends on types of wavelet and its shape. 
It is possible that for choosing wavelet in our application, we can characterize wavelet's frequency band and its central frequency in deferent decomposition levels. Spectra displays of conditions are characterized by distinct frequencies that occur at multiples of a fundamental frequency. When the stress in the wheel exceeds a certain specified magnitude, a sudden recoil movement and subsequent release occurs. This happens periodically and leads to the oscillation of wheels. Depending on the existing frequency, the sound that is thus emitted into the environment is perceived as a short-term noise manifested by unpleasant creaking and wailing. This noise is marked in the paper as a wheel squeal noise. Using the rail track analysis it is possible to define the natural frequencies, the damping ratio or coefficient, and the mode shapes. It is possible to extract sudden changes (singularities) corresponding to vibrations like Daubechies wavelets. Sensors acquire the data and process signals which give many essential parameters for the diagnostic rehabilitation and maintenance of critical urban infrastructure.

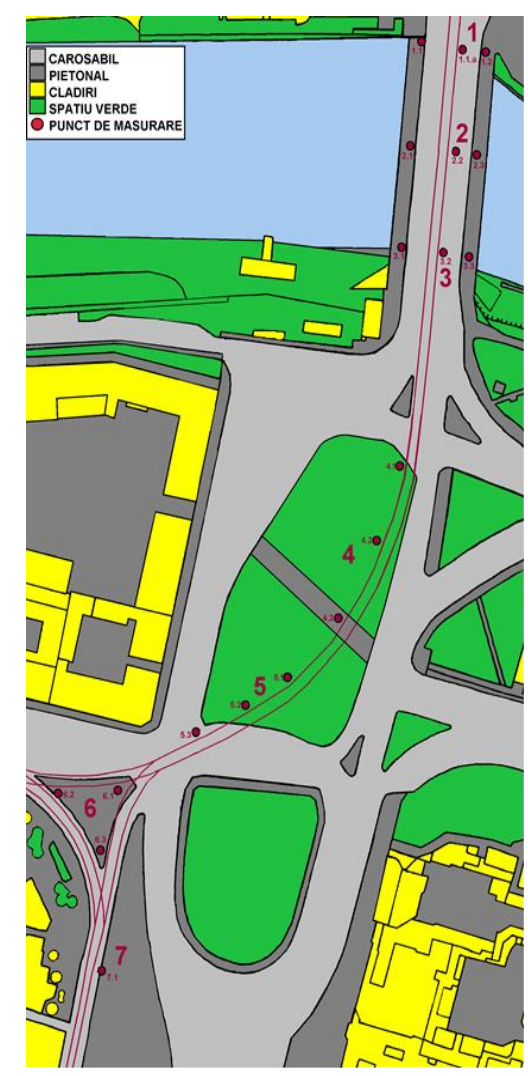

Figure 1. Locations vibration measuring points for Phase II.

In our analysis, we apply a number of signal analytical techniques for time domain decomposition for the identification of the components that may help us to identify whether the tram rail would require a retrofitting or replacement. Material testing is not required if the real time accelerometer data can be used to prove where and when the acquired signal is varying and when the acquired signal is non varying. Based on experimental modal analysis, we also extracted the frequency and mode shape of the structure, since this may be useful for determining and monitoring the condition of the said structure. It is found that the sampling rate for the accelerometer used is the same and has a sampling rate of 10000 Samples/s.

The essence of wavelet packet decomposition is to decompose the high frequency detail signal without decomposition after wavelet transform, so as to improve the frequency resolution. Our analysis is made of three components which are identification of the frequency to extract the material aspect of the noise variation due to tram rail and to identify at the same time the prior covariance the signal is likely to have with the traim rails to distinguish each tram signal and finally to extract the dynamic response of the tram rail against the movement of the tram by the analysis of the spectral representation of the tram rail noise. The time-frequency analyses are able to analyze a non stationary signal wavelet denoising and wavelet packet 
decomposition is an important aspect of research in identification of the eigen frequency that can correspond to the low frequency signal or are some of the more stable signals.

In principle there are two basic approaches to analyze a non stationary vibration signal in time and frequency domain simultaneously. One approach is to split a non stationary vibration signal at first into segments in time domain by proper selection of a window function and then to carry out a Fourier transform on each of these segments separately and deliver an instantaneous spectrum. This is the basic idea for the calculation of the short time Fourier transforms (STFT). Wang has claimed the Short Time Fourier Transform (STFT) to be a powerful tool in detecting local gear damage at an early state [1]. According to one study, STFT will perform better here than conventional methods [2]. Others have investigated STFT methods for the early detection of faults in gears $[3,4]$. While the STFT compromise between time and frequency information can be useful, the drawback is that once you choose a particular size for the time window, that window is the same for all frequencies. Many signals require a more flexible approach - one where we can vary the window size to determine more accurately either time or frequency.

The other approach is the so called wavelet transform (WT), where the non stationary vibration signal to be analyzed is filtered into different frequency bands, which are split into segments in time domain and their frequency contents and energy are analyzed. Wavelet analysis overcomes the disadvantage of STFT since WT uses a windowing technique with variable-sized regions. Wavelet analysis allows the use of long time intervals where we want more precise low-frequency information, and shorter regions where we want high-frequency information. In this study we have applied machine learning based algorithm for feature extraction and source separation problems used to separate useful signal from a mix of signals of interest that remains unvarying with time other than signals and noise. A sudden increase in vibration levels can be an indication of a significant weakening of the structure rail. The travel speed excitation frequency equals the natural frequency of the base-rail-tram system, the system being quasi-resonant mode. We often lack priori knowledge of the structural properties of the tram rail and frequency of vibration induced as time dependent signal. The application of the wavelet packet decomposition is wpdec using dmey wavelet next to calculate wavelet packet decomposition coefficient wpcoef; wavelet packet reconstruction: wprcoef and finally calculate the wavelet packet decomposition energy wenergy using dmey wavelet. This signal recorded is an accelerometer signal is often a mixture of signal noise in tram rail and frequency induced as a result of the tram movement [14]. In the proposed work we have applied a windowing technique for the analysis of the noise spectral distribution series to be transformed is multiplied by a window function before the FFT is done. Spectral characteristics of the noise data amplitude is reasonably reduced during frequency translation [2] and windowing as most of the uncorrelated harmonic data is lost and the power spectral density calculated is lesser than the actual spectral estimation. As we apply the signal to calculate the low noise power generation, there is a chance that when we apply frequency translation, amplitude of the noise for the spectral detection would not work rightly. This is a huge disadvantage in the present scenario and there is a need to extrapolate data terms based on the amplitudes and spectral components of the noise signal data for identification of the conditions of low SNR for sinusoidal signal parameter estimation, for precision and stability of noise parameter enhancement.

The correct selection of the analyzing wavelet with different properties is of critical importance for enhancing the fault features in the wavelet analysis. Various wavelets are available for wavelet applications. Some of them have a good time-frequency localization property which is a desirable attribute for fault detection applications. In this work, four different analyzing wavelets, Morlet, Mexican hat, Discrete Meyer and the Gaussian wavelet were initially examined for their performance in detecting fault conditions. The best performance was obtained from the Complex Morlet wavelet although the Gaussian wavelet also generated good results for the analysis. Therefore, all the results of the wavelet analysis in this study were based on the Complex Morlet wavelet. The advantage of the proposed wavelet in this work is that harmonic frequency can also be easily controlled by the correct selection of F c, and not only the scale parameter a. Consequently, this provides extra flexibility to obtain a good time-frequency resolutionPrior to this technique, noise frequency translation for spectrum representation was increased by coherent gain for noise spectrum identification [3] and a correction factor was applied using Blackman Harris [4] algorithm to identify more number of signal sub segments in the noise signal. The second aspect of the research is to identify the covariance's and classify them for health monitoring and structural health monitoring of tram rails by the identification of the sinusoids in the signal sub space using Eigen value decomposition of the correlation matrix in order to plot the sinusoids in the noise signal data for harmonics. In Blind Signal Separation (BSS) [5] weakness of the prior information is precisely the strength of the BSS model, making it a versatile tool for exploiting the 'spatial diversity' [6] provided by an array of sensors. Promising applications has already been found in the field of processing of communications signals [7]. 


\subsection{Frequency Analysis and Source Signal Separation}

Analysis of the Signal based on the frequency generation is an important aspect of the simulation algorithm designed for the purpose of the tram track designed identifying when it will cross the natural frequency of the guard rails. It is therefore necessary to identify all the components associated with tram tracks to define the procedure for signal separation of the components. It is therefore imperative to define certain rule based features including Independent Component Analysis and likelihood identification that is good in the purpose of solving the problem of signal distinction and frequency components generated as a result of signal vibration in the tram tracks. A sudden increase in vibration levels can be an indication of a significant weakening of the structure rail that can sustain the prevailing shock and vibration caused by discontinuities caused by the impact rails.

\section{VIBRATION BASED ANALYSIS}

The most commonly used data acquisition instruments for vibration-based damage detection are acceleration and displacement transducers. These instruments measure the acceleration or displacement time history signals of a structure and convert them into voltage signals. The mass and size of the data acquisition system must be small compared with the mass of the structure in order to not interfere with the output response. The output dynamic response is usually measured using transducers that measure position, velocity or acceleration. The spectrum identified in the vibration subspace comprises of three important components which are signal which has a finite nature, sinusoids which are signals that are time varying and harmonics which are multiple order coefficients of the noise data. In the noise subspace, application of simple Direct Frequency Translation or periodogram analysis for spectral identification of the power spectral density is of disadvantage in many scenarios and thus there is a need for applying better windowing techniques for a clear spectral representation of the noise power for the noise source. Nevertheless, due to the fact that the signal was filtered, the noise contribution due to spectral clustering techniques in identifying the discontinuities associated with the noise and map the discontinuities on the surface. We also identify the scallop noise [8] due to vibrations to provide an improved method for development of conditions of low SNR for signal parameter estimation.

\subsection{Experimental Results}

We formulate the problem of BSS as one of solving a generalized eigenvalue problem [9], where one of the matrices is the covariance matrix of the observations and the other is chosen based on the underlying statistical assumptions on the sources. This view unifies various approaches in simultaneous decorrelation and ICA, together with PCA and supervised methods using prior probability. Independent component analysis is highly directional and depends on the eigenvalues. The source signals which are extracted as noise and signal of the train are independent and non-gaussian. The typical use of Independent Component Analysis [10] is centering, whitening and dimensionality reduction as pre-processing. The ICA however cannot identify the actual number of source signals but we can ensure that whitening can be done for static signal processing as linear transformation between the signals into independent components measured by some factor of independence. Hence, we need to identify vibrations generated by the running rail is an important component of the noise excitation. We first need to demonstrate that whether for a statistical mixture, the signal satisfies the various statistical assumptions, the different choices for the noise causing the un-mixing of the values. We found that if the damping values are increasing beyond a certain point mixed signal satisfies all the three components of the statistical estimators including non-stationary, non-white and non-Gaussian. The recovered matrix was found to be independent for all values of the noise subspace. Figure 2 shows when the correlation subspace was identified as a matrix that can define the signal detection and isolation for the frequency range and design a scenario for feature extraction of the required points for change in stiffness of the tram rail. These noises are due and existing sources on the vehicle (engines, transmissions and other mechanical components). Obviously, vibrations generated by the running rail is an important component of the noise excitation.

\section{NATURAL FREQUENCY OF THE TRAM RAIL}

The data was taken by a simulated signal where the frequency has been varied from $30 \mathrm{~Hz}-60 \mathrm{~Hz}$ whereby the signal was simulated and an additive noise was added to the signal only to separate the signal from the noise. We first calculate the error variance of the signal and then compute the covariance matrix to calculate the eigen value and eigen vectors using Kurtosis method. Finally, we apply the whitening matrix and apply an orthogonal transform [11] to extract the main signal from the noise vibration. We then subtract the noise from the main data as the noise is additive for non-Gaussian and no-stationarity to identify the

IJEEI, Vol. 6, No. 1, March 2018 : 1 - 11 
noise. This noise is a result of the vibration in the tram lines and this is implemented in the second function to identify the natural frequency of the tram lines.
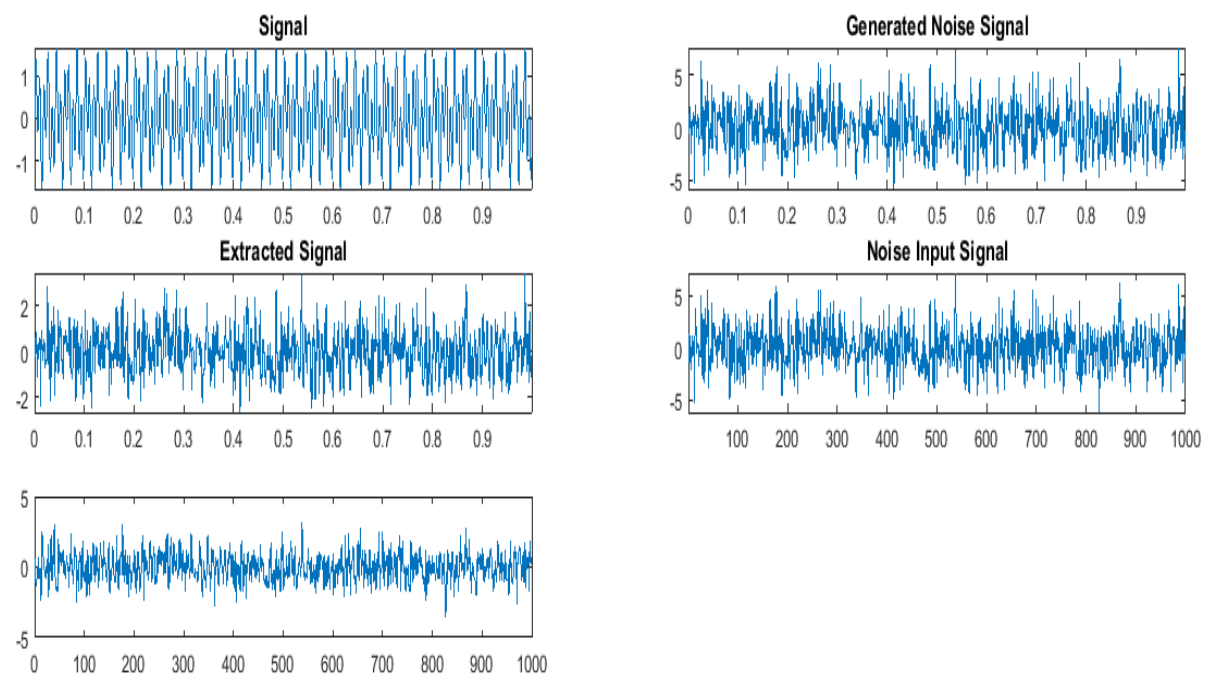

Figure 2. Signal Separation into Tram Movement and Rail Vibration

In order to read signal values directly off the plot; second, to read the noise power spectral density directly off the plot; and third, to quantitatively determine the power in any frequency band by adding the values of all bins in that band. For any number of wavelet stages

$$
D_{j} x(t)=\sum_{n} d_{n}^{j} \varphi\left(2^{j} t-n\right)=\sum_{n} d_{n}^{j} w_{1}\left(2^{j} t-n\right)
$$

can be decomposed into wavelet packet components

$$
D_{j, k, m} x(t)=\sum_{n} d_{n}^{j, k, m} w_{2^{k}+m}\left(2^{j-k} t-n\right) \quad m=0,1, \ldots, 2^{k-1}
$$

of the orthogonal and, of which

$$
D_{j, 0,0} x(t)=D_{j} x(t)
$$

For the signal, if $\mathrm{j}=0$, then $\mathrm{k}=0$ and $\mathrm{m}=0$, the original signal itself at the resolution level $\mathrm{j}$ is denoted by. If the decomposition is $1, \mathrm{k}=1, \mathrm{~m}=0,1$, The packet is decomposed on the first layer to get the decomposition signal and. If the decomposition of 2 , that is, $\mathrm{k}=2, \mathrm{~m}=0,1,2,3$, in the wavelet packet decomposition on the second layer to get the decomposition signal. It obtains the power spectrum curve for each node by first computing the Fast Fourier Transform of the noise data time history signal. If we want to see smaller peaks in the noise floor, then we need not optimize the FFT window. We do not need to measure or simulate a longer data sequence or rather a distribution. Then it multiplies the transformed signal by its complex conjugate and scales the value by the number of samples points contained in the noise time history signal. Once the power spectrum curve is obtained, the algorithm finds its peaks correspond to the natural frequencies of the system. Lastly, output the frequency values (in radians per second) that correspond to the power spectrum curve, fs returns a frequency vector, $f$, in cycles per unit time. An algorithm design for the entire work process is shown below as in Figure 3: for the spectral extraction of signal. A noise spectrum suffers from several disadvantages including loss of the harmonic components due to frequency translation using Direct frequency translation. In order to apply for corrections in windowing before spectrum sensing, we need to achieve either resampling of the noise data characteristics and so windowing before FFT decreases the amplitude compared to the non-windowed data. As the natural frequencies of the signal and the resolution Fs/N (sampling frequency to the number of sample) such that frequencies will be all multiple of 
this resolution and there will be leakage. There is a $1 / \mathrm{N}$ term in the DFT/FFT formula in order to estimate the amplitudes of spectral components of the signal, where $\mathrm{N}$ is the transform length. In case of signal sensing by using FFT, windowing decreases the amplitude of the spectral components. For windowed DFT/FFT, we replaced the average sampling rate $\mathrm{N}$ into a more generalized term for identification of the aggregate of the summation of the windows that correspond to the noise data.

In order to avoid this leakage, we have to develop a methodology as the proper spectral representation based on amplitude of the signal is very important. We add a signal obtained by periodogram to estimate the power spectral density signal s1 and then find the power spectrum corresponding to the maximum variance.

$$
x\left(t_{n}\right)+\epsilon_{n}=c_{i} y\left(t_{n}\right)
$$

The mean square error is given as

$$
\in \mathrm{n}=\sum_{\mathrm{n}=1}^{\mathrm{n}}\left\{\mathrm{x}\left(\mathrm{t}_{\mathrm{n}}\right)-\mathrm{c}(\mathrm{i}) \mathrm{b}_{\mathrm{i}}\left(\mathrm{t}_{\mathrm{n}}\right)\right\} \quad 2
$$

\section{RESULTS}

Wavelet analysis developed in the 1980s could be conducted in time domain and frequency domain analysis for studying the non-stationary signals simultaneously in time as well as frequency domain due to time-frequency localization property of the wavelets. For any number of wavelet stages

$$
D_{j} x(t)=\sum_{n} d_{n}^{j} \varphi\left(2^{j} t-n\right)=\sum_{n} d_{n}^{j} w_{1}\left(2^{j} t-n\right)
$$

Can be decomposed into wavelet packet components

$$
D_{j, k, m} x(t)=\sum_{n} d_{n}^{j, k, m} w_{2^{k}+m}\left(2^{j-k} t-n\right) \quad m=0,1, \ldots, 2^{k-1}
$$

Of the orthogonal and, of which

$$
D_{j, 0,0} x(t)=D_{j} x(t)
$$

For the signal, if $\mathrm{j}=0$, then $\mathrm{k}=0$ and $\mathrm{m}=0$, the original signal itself at the resolution level $\mathrm{j}$ is denoted by. If the decomposition is $1, \mathrm{k}=1, \mathrm{~m}=0,1$, The packet is decomposed on the first layer to get the decomposition signal and. If the decomposition of 2 , that is, $\mathrm{k}=2, \mathrm{~m}=0,1,2,3$, in the wavelet packet decomposition on the second layer to get the decomposition signal and. And so on. The wavelet packet decomposition and reconstruction algorithm is as follows:

1) Will be a signal sequence

$$
s=\{x(k), k \in z\}
$$

Divided into two sub-sequences: even sequences $s_{e}=\left\{s_{e}(k), k \in z\right\}$

$$
\begin{gathered}
\text { Odd sequence } s_{o}=\left\{s_{o}(k), k \in z\right\} \\
\qquad \begin{aligned}
s_{e}(k)=x(2 k) & k \in z \\
s_{o}(k)=x(2 k+1) & k \in z
\end{aligned}
\end{gathered}
$$

$\mathrm{K}$ is the subsequence and the sample number in the subsequence.

2) The respective bands are decomposed by the following formulas

$$
s_{l\left(2^{l}-1\right)}=s_{(l-1) 2^{l-1} o}-p\left(s_{(l-1) 2^{l-1} e}\right)
$$




$$
S_{l 2^{l}}=S_{(l-1) 2^{l-1} e}+u\left(s_{l\left(2^{l}-1\right)}\right)
$$

3) The reconstruction process of the wavelet packet is to keep the corresponding band signal, and the other band signal is set to zero, and then reconstructed according to the following way.

$$
\begin{array}{cc}
s_{(l-1) 2^{l-1} e}=s_{l 2^{l}}-u\left(s_{l\left(2^{l}-1\right)}\right) & \\
s_{(l-1) 2^{l-1} o}=s_{l\left(2^{l}-1\right)}+p\left(s_{(l-1) 2^{l-1} e}\right) & \\
S_{(l-1) 2^{l-1}}(2 k)=s_{(l-1) 2^{l-1} e}(k) & k \in z \\
S_{(l-1) 2^{l-1}}(2 k+1)=s_{(l-1) 2^{l-1} o}(k) & k \in Z
\end{array}
$$

\subsection{Wavelet energy decomposition}

As a result of wavelet packet decomposition, the decomposition of the band independent of each other, the information is not redundant, nor omit, the energy balance.

The energy $\mathrm{E}(\mathrm{n})$ in the wavelet packet is

$$
E(n)=\sum_{k=1}^{N}\left|g_{n}(k)\right|^{2} \quad n=0,1,2, \ldots, 2^{m}-1
$$

The structure of wavelet packet transform is very similar to DWT. The difference is that in the DWT, only the approximation coefficients are decomposed while in the wavelet packet transform, both the detail and approximation coefficients are decomposed. Therefore, WPT offers a more complex and flexible analysis. A 'n'level wavelet packet decomposition produces $2 \mathrm{n}$ different sets of coefficients as opposed to $\mathrm{n}+1$ sets in the DWT where $\mathrm{n}$ is the band number. A single decomposition using wavelet packet transform generates a large number of wavelet bases. From the wavelet bases are being used to represent the information contained in the time series of interest and hence should mimic its underlying features. We decompose a data at depth 3 (level 3) with db4 wavelet using default entropy (Shannon). We obtain a complete binary tree, wavelet tree, and best tree of a time series data. From the tree values we calculate descriptive statistics at various level packets. It will represent the characteristics of time series. Due to this time frequency localization property, the wavelet transform gives better decomposition of signal in spectral domain than the conventional Fourier transform or windowed Fourier transform. Wavelet transform uses wavelength adaptive convolution operators that are optimal on the basis of wavelength of the studied portion of a signal.

Through using wavelets, variations in frequency components with time can be observed. We have used the CWT to obtain a time-frequency analysis of a 1-D signal using an analytic wavelet with cwt. Vibration signals where sudden changes are recorded as transients are suitable where the CWT provides similar results to the STFT and an example where the CWT can provide more interpretable results than the STFT. Finally, it is possible to reconstruct time-scale (frequency) localized approximations to a signal using icwt. The wavelet transform is particularly suitable for non-stationary signals. Wavelet transform is a perfect way to analyze non-stationary signal for its multi-resolution nature as by varying the width of the mother wavelet, the time-frequency resolution at the largest scale can be varied, which in turn determines how the resolution changes as the frequency increases at shorter scales.

This is useful for monitoring some critical frequency components to the performance of the structure. In contrast to the Fourier transform, the wavelet transform allows exceptional localization, both in the time domain via translation $t$ of the wavelet, and in the frequency domain via dilations scales b, which can be changed from minimum to maximum, chosen by the user. Wavelet method are used for localized characteristics whereby duration the phase in signal magnitude and the analyzed for localized characteristics and simulates waves for non- stationary random processes whereby the study is done based on factors such as localized contours observed in each scalograms are imprints of seismic perturbation on radon time series data as they satisfy the criteria laid down in the governing empirical relation that is $\mathrm{D}<\mathrm{Rmax}$. Wavelets can filter out the noise without removing the spikes that characterize changes in water levels prior to earthquakes. Adding up the squared co-efficient within each level, one can measure the energy content of each scale, similar to the power spectral density used in Fourier analysis. But the earthquake gestation process is a nonlinear process having non-stationary time series vibration data sequence, which carry different at different times of retrofitting information. 
Fourier transform, maximum entropy spectral analysis methods are based on steady-state signal based on the spectral analysis method, the overall signal is given only contains various frequency components. Windowed Fourier transform it is only suitable for analysis of all the single characteristic scales roughly the same process although with a certain time-frequency domain features as it's time - frequency window size is fixed. Vibration wave characterization is essential for better understanding wave propagation phenomena and the description of the local effects produced by the radon distribution.

Wavelet transform reveals the seismic activity cycle time variability, revealing the nonlinear process of seismic activity. The effect appears as a transient oscillation with a 5- to 10-day period, which is initiated a few days before a large earthquake and decays over a few days to weeks after it. The wavelet transform to make up traditional Fourier transform spectral analysis method is less time-frequency domain, complex wavelets have good frequency resolution, but have constant power across the time duration of an oscillation, which does not enable extrema to be detected. Time-frequency signal analysis of earthquake records using Mexican hat wavelets and Morlet wavelets. For real wavelets, such as the Mexican Hat wavelet, phase and amplitude information is superposed in the time-frequency plane; they are useful for detecting extrema and discontinuities (Serroukh et al., 2000). The Morlet spectrum shows a narrow frequency band of high power centered at $7 \mathrm{~s}$, but the amplitude of the signal is constant for the duration of the signal. In order to explore fully the behaviour of wavelets we thus investigate two smooth wavelets with very different features: the complex Morlet wavelet and the real Mexican Hat wavelet whereby mother wavelet satisfies the following two conditions.

$$
g(f)=2 \cos ^{l}(\pi f) \sum_{l=0}^{l / 2-1} l / 2-1+l \sin ^{2 l}(\pi f)
$$

We define the (continuous) wavelet transform of a signal $x(t)$ as

$$
T_{x}(t, a ; \varphi)=\int_{-\infty}^{\infty} x(s) \varphi_{t, a}^{x}(s) d s
$$

where $\varphi_{t, a}(s)=1 / \sqrt{a} \varphi_{0}(s-t) / a$ and $\varphi_{0}$ is a fixed function, called the mother wavelet, which has zero mean and is well localized in time and frequency. There are clearly an infinite variety of functions fulfilling these criteria; one commonly used wavelet is the Morlet wavelet, given by $\varphi(\eta)=\pi^{1 / 4} e^{-1 / 2 \eta^{2}} e^{i 6 \eta}$ which is the wavelet we will use in this section as it has excellent frequency resolution for a certain bandwidth. Hence by analyzing the coefficients in a particular band represent the energy at time intervals equally spaced over the duration of the signal. When the squared coefficients are plotted on a time-scale grid, the transfer of energy from one band to the next may be observed along the time scale. This is called the scalogram or mean square map. The volume bounded by the surface is the mean square value of the signal. The proposed signal processing methodology for scalogram can be used to investigate the characteristics of variance in radon data recorded on various types of sites and their effects on different types of structures. The scalograms reflect localized contours which are present as time dependent frequency fluctuations are the signatures of the anomalies embedded in the radon time series data recorded simultaneously even for low frequencies where we use a very wide window for better frequency resolution. For real wavelets, such as the Mexican Hat wavelet, phase and amplitude information is superposed in the time-frequency plane; they are useful for detecting extrema and discontinuities (SWP2000). The simulated seismic history is obtained based on the response spectrum and the earthquake correlation process. It has a good localized function where $\mathrm{a}(\mathrm{t})=$

$$
\sum_{k=0}^{n} C_{k} \cos \left(\varpi_{k} t+\varphi_{k}\right)
$$

The Morlet wavelet translation method has been used to analyze the dynamic period of some area of vibration data. The results show that there exist both comparatively stable periods and some variable periods with time. We use the recent periods and more stable period to predict the tendency for analysis wavelets such as the Morlet wavelet reaches a maximum at the instantaneous frequency Á0(t). 

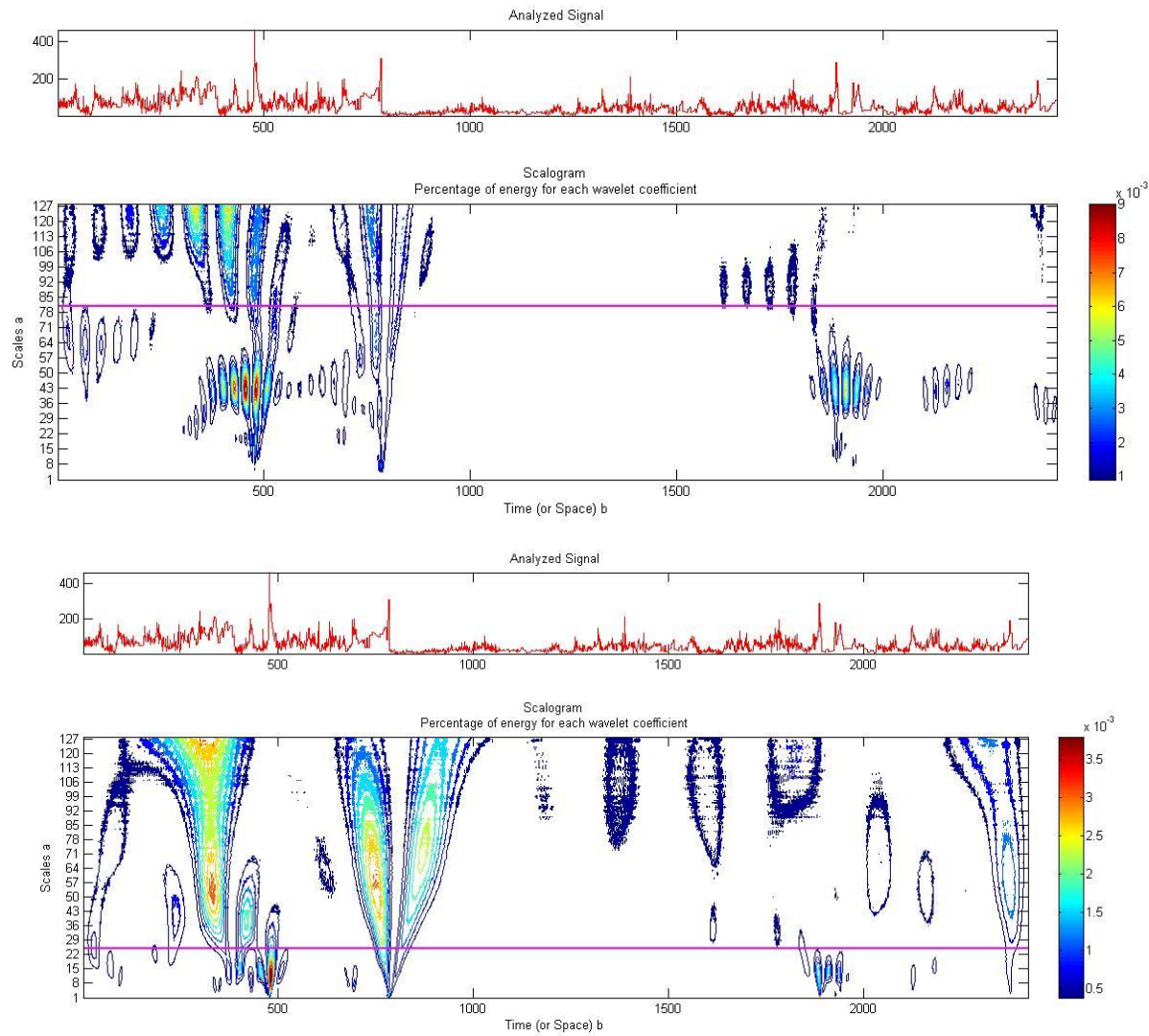

Figure $4 \mathrm{a}$ and b. Morlet (middle) and Mexican Hat (bottom) scalograms of vibration signal (top)

The variations of the frequencies corresponding to the maximum of the wavelet transform at particular time are very similar to the recorded frequencies. Linear and nonlinear statistics to the recorded long-term time series data are absolutely essential for bringing out the information apparently unseen in the precursory signals. Percival (1995) showed that the time integral of the wavelet scalogram can be used as an unbiased, consistent estimator for the power spectrum, thus for practical purposes the wavelet scalogram satisfies the marginal properties.

$$
\begin{aligned}
& A_{\text {scalo }}(t, a)=\left|T_{x}(t, a ; \varphi)\right|^{2} \\
& A_{\text {scalo }}(t, a)=\int_{-\infty}^{\infty} \int_{-\infty}^{\infty} W_{x}(\mathrm{~s}, \xi) \mathrm{W}_{\varphi}\left(\frac{\mathrm{s}-\mathrm{t}}{a}, \mathrm{a} \xi\right) d s d \xi
\end{aligned}
$$

The single point source displacement of ground motion is idealized by a Gaussian function. For the purpose of signal analysis of the radon count data generated by a simple point source rupture is used to form the basis wavelet function. The result of the signal processing of radon data count is presented in the form of a scalogram using the coefficients of the continuous Mexican hat wavelet or Mortlet wavelet transform can describe the signal energy in the time-scale domain. The maximal ridges in the Morlet spectrum show both periodicities, although there is a small oscillation in the estimated period for both periodicities. The maximal ridges in the mexican hat wavelet analysis identify the maxima and minima in the signal at the correct times, but the periods are not correctly resolved; there is only a single line of maxima points, fluctuating around a period of about $500 \mathrm{~s}$. 


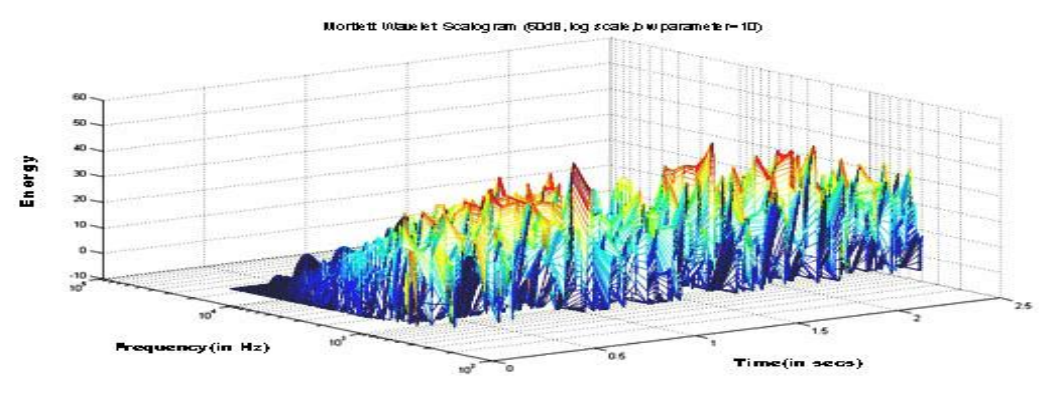

Figure 5. Identification of wavelet ridge using the mortlet wavelet transform for percentage of energy with identical coeffcients

\section{CONCLUSION}

Our work is a useful tool for the purpose of defining the process of signal noise extraction and noise power spectral density measurement from the vibrating rails. As we are using compression type accelerometer which is more sensitive to the zero shift in the impact test, it is not possible to get correct signal, and thus cannot obtain the correct velocity, displacement using common integration approach as well. It can be said that the velocity and displacement shows unrealistic trend to increase continuously without approaching zero.

We have developed analytical expressions to find the performance of the estimate and we have corroborated this study applying Independent component analysis based on signal separation and information detection for the estimation of the power spectral density for the spectrum of the tram data. We have done a computational approach to observe the spectrum signal components for frequency ranges higher than (300 $\mathrm{Hz}$ ) for vibration caused by the rolling of wheels on rails and resonance wheels caused by frictional forces are minor and occur only sporadically compared to the vibrations of low frequency generated type of impact forces. In particular, we have taken measurements along 7 spots along the Oradea city for each different type of tram and identified the nature of the power spectral density and frequency content of the noise which corresponds to the tram rail vibration.

Estimation of the signal characteristics for noise is a difficult proposition as most of the signal spectra including the noise amplitude due to the sinusoids and harmonics are lost due to signal averaging when we apply frequency translation. In order to better estimate the power spectral density for the associated tram rail measurement for the accelerometer, we apply a number of machine learning approaches which gives us a better spectral estimation. Our theoretical predictions for non- linear spectral estimation for noise were confirmed experimentally using simulated and real data acquired signals. We were also able to apply a parametric signal processing for the extraction of the frequency of the rail vibration when the tram passes the rail and compare with the actual data. In place of resampling the noise data, we applied a Welch correction factor to reduce distortions to correct the amplitude of spectral components.

We have found a periodic behavior of the noise spectrum, which corresponds to the frequency of the mean sampling period. For higher frequency, auto-regressive estimators behave better that linear resampling, but the estimation with parameter estimation approaches are better than the FFT as the mean noise frequency increases. We have also designed the methodology for identification of the power spectrum energy loss during windowing. We tested for all samples and identified the output. As the plausible approach, we find that we were able to enhance the output noise signal by applying a summation of all components of the noise signal than frequency averaging using a Direct frequency translation and the mean square error is lower. Further using a Blackman Harris power correction, we increased the resolution of the sub segments of the signal before normalizing them or applying the Welch Function. This approach is suitable in the future in identifying signals where signal separation is difficult and identification of the upper noise level is a difficult approach. to identify cross correlations to identify the eigen vectors in the signal sub-space and identify the coefficients from the correlation matrix and design a band pass filter in future works around the given input frequency that are more than $3 \%$ above or below the band-pass frequency will be attenuated. 


\section{ACKNOWLEDGEMENTS}

The paper published has been sponsored under the Erasmus Mundus partnership program agreement vide number 2014-0855/001-001 coordinated by and between University of Oradea and City University of London Under Action Plan 2 for the year 2016 for Post-Doctoral Fellowship Programme under the Supervision of Prof Dr Radu Tarca from University of Oradea. This work was conducted with the guidance from Prof. Tiberiu Vesselenyi with report Phase II and data acquired as part of the service contract between RA OTL.

\section{REFERENCES}

[1] R.N. Boubela, K. Kalcher, W Huf., C. Kronnerwetter, P. Filzmoser, and E. Moser, "Beyond noise: using temporal ICA to extract meaningful information from high-frequency fMRI signal fluctuations during rest". Frontiers in human neuroscience, 7, 2013, p.168.

[2] S.V. Vaseghi, "Advanced signal processing and digital noise reduction". Springer-Verlag, 2013.

[3] F. J. Harris, "On the use of windows for harmonic analysis with the discrete fourier transform", In: Proceedings of the IEEE, Jan. 1978, vol. 66, no. 1, pp. 51-83.

[4] M.Belkin, L.Rademacher, and J.R.Voss, “ Blind Signal Separation in the Presence of Gaussian Noise”. In: COLT, 2006,(pp. 270-287).

[5] R. Gribonval, and S. Lesage, "A survey of sparse component analysis for blind source separation: principles, perspectives, and new challenges”. In: ESANN'06 proceedings-14th European Symposium on Artificial Neural Networks (pp. 323-330). d-side public 2006, April.

[6] J.F.Cardoso, “ Blind signal separation: statistical principles.” In: Proceedings of the IEEE, 86(10), pp.2009-2025, 1998.

[7] R.G. Lyons, "Understanding digital signal processing." Pearson Education, 2010.

[8] L.Parra, and P.Sajda, " Blind source separation via generalized eigenvalue decomposition." Journal of Machine Learning Research, 4(Dec), pp.1261-1269, 2003.

[9] P. Comon," Independent component analysis.” Higher-order statistics, pp.29-38, 1992.

[10] P.Comon, "Independent component analysis, a new concept?." Signal processing, 36(3), pp.287-314, 1994.

[11] P. Stoica, and R.L. Moses, "Introduction to spectral analysis.: (Vol. 1, pp. 3-4). Upper Saddle River: Prentice hall, 1997.

[12] E.C. Ifeachor and B.W. Jervis,” Digital signal processing: a practical approach.” Pearson Education, 2002

[13] P. K. Dutta, and O.P.Mishra, "Analysis of zero-crossing frequency and likelihood function for retrieval of maximum displacement in real time earthquake signal," Acta Technica, 2016(To be Published).

[14] Lawrence, T G, Dakin, J M, Norris, P, Forni, M S, Noise and vibration from road and rail RP773 ISBN: 978-086017-693-0, 2011.

[15] OECD Glossary of Statistical Terms, Pages: 602 ISBN:9789264088023, DOI: 10.1787/9789264055087-en, 2006. 